\title{
The logistic-X family of distributions and its applications
}

\author{
M. H. Tahir, Gauss M. Cordeiro, Ayman Alzaatreh, M. Mansoor, M. Zubair \\ Department of Mathematics
}

\section{Abstract}

The logistic distribution has a prominent role in the theory and practice of statistics. We introduce a new family of continuous distributions generated from a logistic random variable called the logistic$\mathrm{X}$ family. Its density function can be symmetrical, left-skewed, right-skewed, and reversed-J shaped, and can have increasing, decreasing, bathtub, and upside-down bathtub hazard rates shaped. Further, it can be expressed as a linear combination of exponentiated densities based on the same baseline distribution. We derive explicit expressions for the ordinary and incomplete moments, quantile and generating functions, Bonferroni and Lorenz curves, Shannon entropy, and order statistics. The model parameters are estimated by the method of maximum likelihood and the observed information matrix is determined. We also investigate the properties of one special model, the logistic-Fréchet distribution, and illustrate its importance by means of two applications to real data sets.

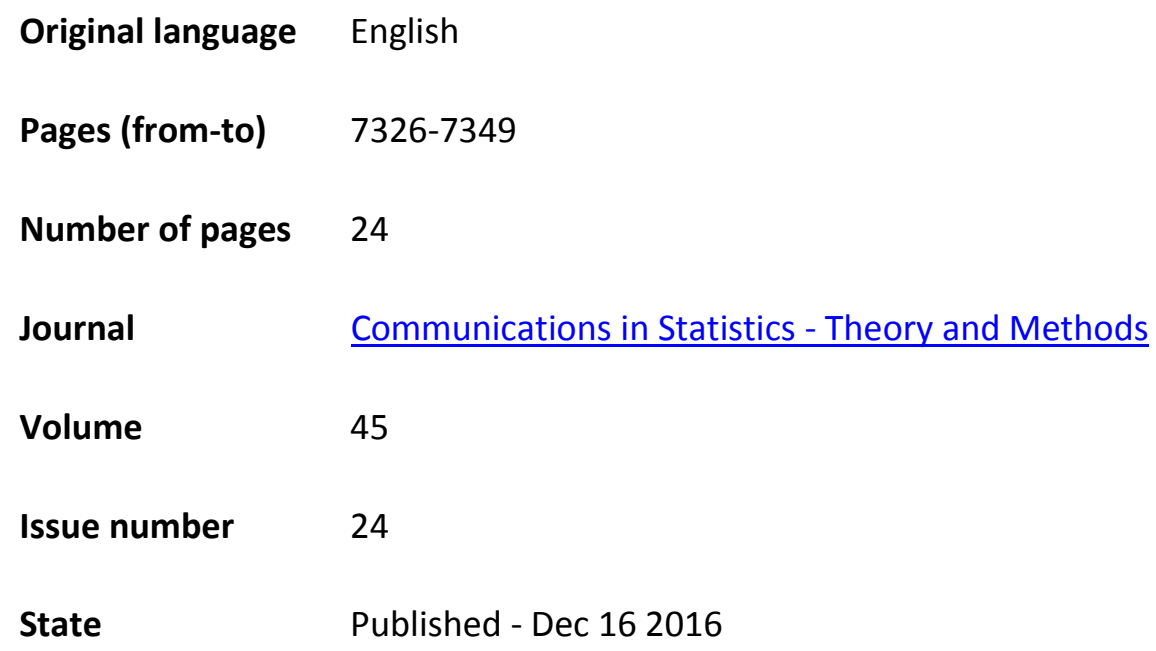

Tahir, M. H., Cordeiro, G. M., Alzaatreh, A., Mansoor, M., \& Zubair, M. (2016). The logistic-X family of distributions and its applications. Communications in Statistics - Theory and Methods, 45(24), 7326-7349. DOI: 10.1080/03610926.2014.980516 